\title{
Varia
}

\section{International Society of Psychoneuroendocrinology}

Preliminary Notice

The 8th International Congress of the International Society of Psychoneuroendocrinology will be held at the Atlanta Hüton Hotel, Atlanta, Ga., May 8-12, 1977. Registration will be on Sunday, May 8. For information please write to Dr. Richard P. Michael, Department of Psychiatry, Emory University School of Medicine, Atlanta, GA 30322 (USA). 